Received May 8, 2020

Revised July 3, 2020

Accepted July 6, 2020

\section{Invisible perforation during an endoscopic procedure of the esophagus under general anesthesia - A case report -}

\author{
Hyun Joo Heo, Ji Hye Lee, Yu Yil Kim, Seung Min Baek, \\ Ki Man Kim, and Da Wa Jung
}

Department of Anesthesiology and Pain Medicine, Presbyterian Medical Center, Jeonju, Korea

\begin{abstract}
Background: Endoscopic procedures of the esophagus are more complicated than those of other regions of the gastrointestinal tract. They have a relatively long procedure time and high risk of complications, such as perforation and bleeding. Perforations that occur during the procedure can accompany pneumoperitoneum and pneumomediastinum through leakage of insufflation air and cause severe ventilatory impairment.
\end{abstract}

Case: A 58-year-old male patient underwent enucleation of leiomyoma in the esophagus using endoscopy under general anesthesia. Ventilatory impairment occurred 15 min after commencement of the procedure. Subsequently, subcutaneous emphysema and severe abdominal distension were observed. We suggested the possibility of microperforation during the procedure to the endoscopist, and he performed endoscopic clipping around the excision site of leiomyoma.

Conclusions: Providing anesthetic care by anesthesiologists during endoscopic procedures is considered necessary for patient safety. Complications of endoscopic procedures can be detected and managed early without sequelae during anesthetic care.

Keywords: Endoscopy; Esophagus; General anesthesia; Perforation; Pneumomediastinum; Pneumoperitoneum.

\author{
Corresponding author \\ Ji Hye Lee, M.D. \\ Pedicine, Presbyterian Medical \\ Center, 365, Seowon-ro, Wansan-gu, \\ Jeonju 54987, Korea \\ Tel: 82-63-230-1591 \\ Fax: 82-63-230-8919 \\ E-mail: 02ljh@hanmail.net
}

Endoscopic procedures (EPs), such as endoscopic mucosal resection, endoscopic submucosal dissection (ESD), peroral endoscopic myotomy, endoscopic submucosal tunnel resection, and endoscopic enucleation, are widely used instead of surgical treatment in early cancerous or other benign lesions in the gastrointestinal tract. As precise and complex manipulations are possible with these EPs, this treatment can be applied to lesions of various sizes and regions of the gastrointestinal tract, including the esophagus [1-3].

EPs are mostly performed under sedation to reduce the anxiety and discomfort of the patient during the procedure and to minimize patient movement for a safe and effective procedure. In gastric ESD, sedation is considered safe and effective $[4,5]$. However, esophageal EPs require moderate to deep sedation or general anesthesia since they are technically difficult compared to gastric or colonic EPs. This is because of the anatomical features of the esophagus, lon-

This is an Open Access article distributed under the terms of the Creative Commons Attribution Non-Commercial License (http://creativecommons.org/licenses/by-nc/4.0) which permits unrestricted non-commercial use, distribution, and reproduction in any medium, provided the original work is properly cited.

Copyright (C) the Korean Society of Anesthesiologists, 2020 
ger procedural time, and higher risk of complications, such as perforation and bleeding [6]. Recently, studies on the safety and efficacy of general anesthesia during ESD reported that general anesthesia can reduce EP-related complications [7]. Deep sedation has a risk of respiratory complications, such as respiratory depression and regurgitation with/without aspiration $[5,8]$. Abdominal distention caused by insufflation air, which is continuously used to maintain the field of view, can exacerbate these respiratory problems in EP. In addition, a perforation that is unrecognized, particularly by the endoscopist, can cause pneumoperitoneum and/or pneumomediastinum and can further aggravate respiratory problems. When these problems occur, early detection and management are important. However, if a procedure is performed without the anesthesiologist who induced sedation, monitoring the condition of the patient, detection of perforation and its management may be delayed, leading to serious complications.

Here, we report a case of severe ventilatory impairment caused by pneumoperitoneum and pneumomediastinum that occurred during enucleation of esophageal leiomyoma under general anesthesia.

\section{CASE REPORT}

This case was approved by the Institutional Review Board of the present hospital (no. E2020-027).

A 58-year-old male patient $(165 \mathrm{~cm} / 71.4 \mathrm{~kg})$ was admitted for further evaluation and management of a submucosal lesion in the distal esophagus. In esophagogastroduodenoscopy, approximately 2-cm-sized ovoid smooth elevation was observed in the cardia of the distal esophagus. The lesion was diagnosed as a leiomyoma $(29 \times 13.6 \mathrm{~mm})$ on endoscopic ultrasound. The endoscopist planned enucleation of the leiomyoma by endoscopic submucosal tunnel resection (ESTR) and requested for general anesthesia for the procedure.

The patient had no specific past history, and physical examination was unremarkable. In addition, no abnormal findings were observed in the chest radiograph, electrocardiogram, or laboratory tests. ESTR was performed in the operation room under general anesthesia by an anesthesiologist. Noninvasive blood pressure, electrocardiography, oxygen saturation $\left(\mathrm{SpO}_{2}\right)$, and bispectral index were monitored. Vital signs before anesthesia were as follows: blood pressure (BP), 169/97 mmHg; heart rate (HR), 58 beats/min; and $\mathrm{SpO}_{2}, 98 \%$. General anesthesia was induced with propofol, remifentanil, and rocuronium, and maintained with desflurane and $\mathrm{O}_{2}$-air mixture $\left(\mathrm{FiO}_{2}\right.$ 0.5). Mechanical ventilation was provided with a tidal volume of $500 \mathrm{ml}$ and respiratory rate of 10 breaths $/ \mathrm{min}$. End-tidal $\mathrm{CO}_{2}\left(\mathrm{EtCO}_{2}\right)$ and peak inspiratory pressure (PIP) were $31 \mathrm{mmHg}$ and $20 \mathrm{cmH}_{2} \mathrm{O}$, respectively.

After $15 \mathrm{~min}$ from the beginning of the procedure, PIP suddenly increased to $35 \mathrm{cmH}_{2} \mathrm{O}$, tidal volume decreased to 80-100 ml, and $\mathrm{SpO}_{2}$ reduced to $96-94 \%$. Immediately, we explained the condition of the patient to the endoscopist and stopped the procedure. We performed manual ventilation and auscultation. On auscultation, the right lung sound was weak, and the left lung sound was inaudible. We checked the position of the endotracheal tube using fiberoptic bronchoscopy to confirm the possibility of one-lung ventilation. The endotracheal tube tip was located $2 \mathrm{~cm}$ above the carina, and no secretion was found in the bronchus. Abdominal distension of the patient was observed; however, obvious perforation was not found during endoscopic evaluation. Therefore, we considered that abdominal distension was because of the expansion of the gastrointestinal tract caused by insufflation air used during the procedure. BP (110-130/70-90 $\mathrm{mmHg}$ ) and HR (50-60 beats/min) were stable, and PIP ( $\left.30 \mathrm{cmH}_{2} \mathrm{O}\right)$, tidal volume (300-350 ml), and $\mathrm{SpO}_{2}(96 \%)$ recovered and were maintained while recruitment was conducted through manual ventilation. Subsequently, ventilation was adjusted to PIP of $29-32 \mathrm{cmH}_{2} \mathrm{O}$ and respiratory rate of 13-14 breaths/min in the pressure control mode. Further, $\mathrm{EtCO}_{2}$ was maintained at $35-41 \mathrm{mmHg}$. We decided to resume the EP. After approximately $10 \mathrm{~min}$, subcutaneous emphysema was palpated in the upper chest and neck, and abdominal distension had aggravated. Therefore, we notified the possibility of air leakage due to perforation in the field of procedure to the endoscopist; however, the identified perforation was not located. We suggested the possibility of microperforation, and the endoscopist used six endoscopic clippings to repair the suspected perforation after removing the leiomyoma and terminated the procedure (Fig. 1). Neuromuscular blocks were reversed, and extubation was performed after consciousness fully recovered. Vital signs upon arrival at the postanesthetic care unit were as follows: $\mathrm{BP}, 174 / 84 \mathrm{mmHg}$; $\mathrm{HR}, 88$ beats/min; $\mathrm{SpO}_{2}, 92 \%$; and $\mathrm{O}_{2}, 5 \mathrm{~L} / \mathrm{min}$ via a facial mask. The patient complained of discomfort and abdominal pain during respiration. Severe abdominal distension was observed, and subcutaneous emphysema spread throughout the eyelid, neck, chest, and abdomen. Pneumoperitoneum, pneumomediastinum, and subcutaneous emphysema were verified on portable chest 
X-ray (Fig. 2). The endoscopist performed paracentesis using a 14-gauge angio-needle to remove air from the peritoneal cavity, but it failed. We decided to transfer the patient to the intensive care unit until the patient was stable. No signs of leakage were detected on esophagography 2 days after the procedure. The patient underwent conservative treatment and was discharged without complications.

\section{DISCUSSION}

EPs are economical, noninvasive, and less fatal compared to surgical procedures; therefore, they are commonly
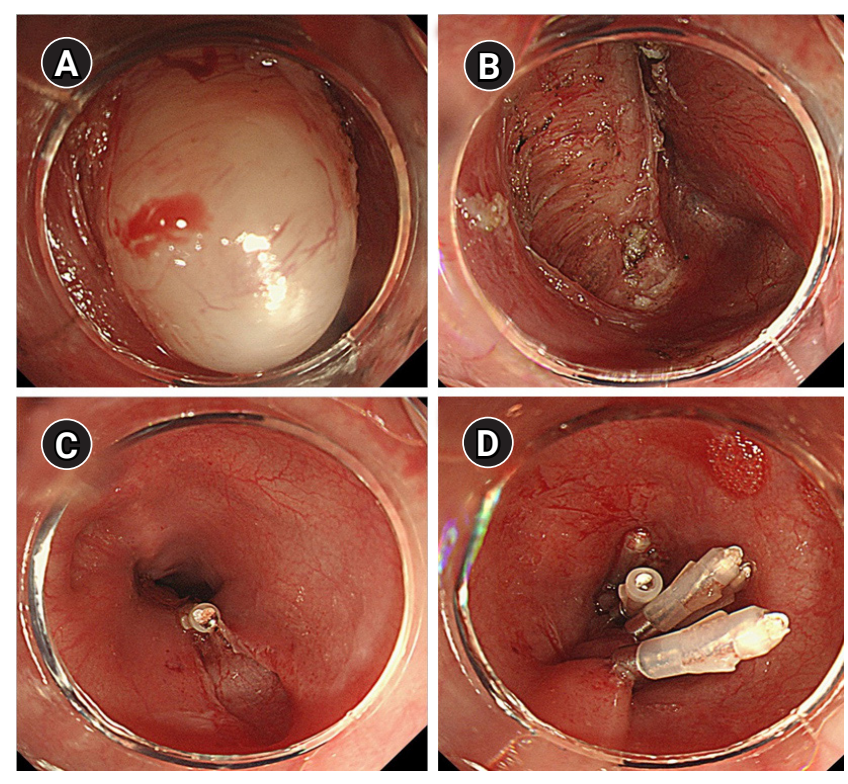

Fig. 1. Endoscopic submucosal tunnel resection. (A) Submucosal esophageal leiomyoma. (B) Submucosal space after removal of leiomyoma. (C and D) Clipping of the esophageal mucosa. used for the management of early cancerous lesions of the gastrointestinal tract. In addition, precise and complex manipulation is possible with the development of various techniques related to EP. Moreover, en-bloc resection is possible using EPs for cancerous and large benign lesions. Therefore, indications of EPs are extensive.

The precise and complex manipulation of EP involves a lengthy procedural time and requires minimum patient movement for a safe and efficient procedure [7]. Therefore, sedation is usually required during the procedure. In particular, the esophagus is anatomically narrow, curves, and has no serous membrane. It is affected by cardiac and respiratory movements because of its location [9]. Therefore, esophageal EPs are technically more complicated, require a longer time, and have more complications, such as perforation and bleeding, compared to other regions of the gastrointestinal tract. As a result, patients particularly require moderate or deep sedation or general anesthesia during the procedure [6]. For safety and effectiveness of the procedure in this patient, we selected general anesthesia for the following reasons: first, the patient's lesion was a leiomyoma, which is a submucosal tumor, and was relatively large in size $(29 \times 13.6 \mathrm{~mm})$. Second, enucleation of the leiomyoma by ESTR is more complicated and elaborate, and involves a longer procedural time than ESD.

Gastric perforation caused by an injured muscle in EPs is confirmed by a fistula. Perforation in the abdominal cavity or greater omentum in the endoscopic view and is confirmed by free air in the abdominal cavity with a simple abdominal X-ray or subcutaneous emphysema. However, it is difficult to confirm esophageal perforation and muscle lay-
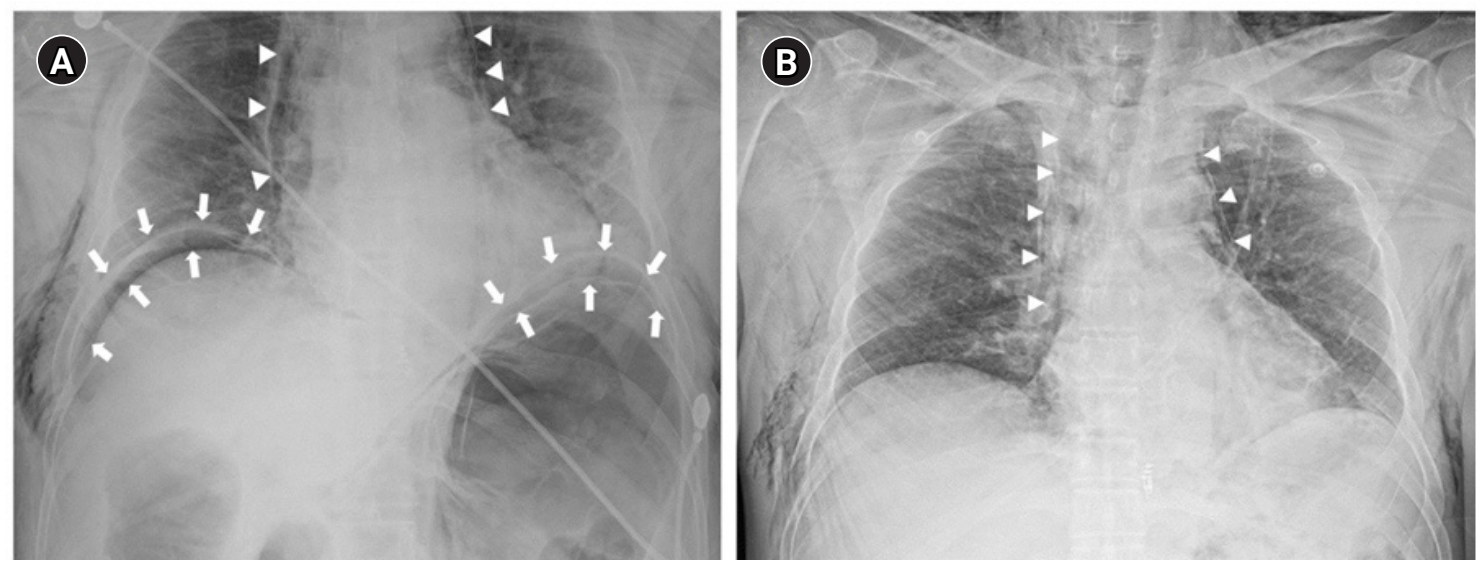

Fig. 2. Chest radiographs. (A) Endoscopic submucosal tunnel leiomyoma resection shows pneumoperitoneum (arrows), pneumomediastinum (arrowheads), and generalized subcutaneous emphysema. (B) On postprocedural day 1, pneumoperitoneum has disappeared, but pneumomediastinum (arrowheads) and subcutaneous emphysema have persisted. 
er laceration because the esophagus is located in the mediastinum. Undetected microperforation or unrecognized perforation by an endoscopist in esophageal EPs may cause severe pneumoperitoneum and/or pneumomediastinum by continuous leakage of insufflation air used during the procedure. Furthermore, increased abdominal pressure causes elevation of the diaphragm, limited tidal volume, decreased venous return, and decreased cardiac output. Conscious patients may complain of discomfort, but the detection of symptoms and signs, and further management may be delayed for sedated or anesthetized patients.

In this case, while excising the submucosal leiomyoma of the lower esophagus during the $\mathrm{EP}, \mathrm{SpO}_{2}$ suddenly decreased with an abrupt increase in PIP and decrease in tidal volume. On fiberoptic bronchoscopy, it was observed that the position of the endotracheal tube was normal, and there was no airway obstruction by secretion. In addition, the endoscopist confirmed that esophageal perforation was not visible with the endoscope. However, prominent pneumoperitoneum, pneumomediastinum, and subcutaneous emphysema that presented on chest X-ray after the procedure were evidences of perforation during the procedure. This indicated that a microperforation may have occurred that was not recognized by the endoscopist. As the patient's respiration and vital signs were continuously monitored by the anesthesiologist, ventilatory impairment with subcutaneous emphysema was immediately detected. Subsequently, the anesthesiologist stopped the procedure and notified the endoscopist regarding the possibility of esophageal perforation. Therefore, the endoscopist could perform clipping in the area of suspected perforation immediately. If there was no anesthetic care during procedure in the endoscopy unit, microperforation would have been detected after severe hemodynamic dysfunction or termination of the procedure. Consequently, additional treatment, including surgical management, could be required because of delayed detection [10].

An endoscopist who is performing the EP may be unaware of complications, such as microperforation, without bleeding or massive injury in the field of the procedure. Additionally, early management of complications may be insufficient without a trained person, such as an anesthesiologist, for sedated patients. Patient management by an anesthesiologist can reduce the mortality and surgical treatment rate via rapid detection and treatment during the intraprocedural period when complications occur in EPs $[7,10]$.

In conclusion, EPs are broadly applicable complex pro- cedures, and therefore, require deep sedation or general anesthesia. Anesthetic care by the anesthesiologist is considered necessary for patient safety, and complications related to the EP can be quickly detected and treated without undesirable sequelae during anesthetic care. In addition, the anesthesiologist should understand the process of the $\mathrm{EP}$ and its complications.

\section{CONFLICTS OF INTEREST}

No potential conflict of interest relevant to this article was reported.

\section{AUTHOR CONTRIBUTIONS}

Conceptualization: Hyun Joo Heo. Data acquisition: Seung Min Baek, Ki Man Kim, Da Wa Jung. Supervision: Ji Hye Lee. Writing_original draft: Yu Yil Kim. Writing-review \& editing: Ji Hye Lee.

\section{ORCID}

Hyun Joo Heo, https://orcid.org/0000-0003-3269-3844

Ji Hye Lee, https://orcid.org/0000-0003-2507-6629

Yu Yil Kim, https://orcid.org/0000-0003-3455-9251

Seung Min Baek, https://orcid.org/0000-0003-0290-0208

Ki Man Kim, https://orcid.org/0000-0002-4257-9167

Da Wa Jung, https://orcid.org/0000-0002-1566-7903

\section{REFERENCES}

1. Gotoda T, Yamamoto H, Soetikno RM. Endoscopic submucosal dissection of early gastric cancer. J Gastroenterol 2006; 41: 92942.

2. Chung IK, Lee JH, Lee SH, Kim SJ, Cho JY, Cho WY, et al. Therapeutic outcomes in 1000 cases of endoscopic submucosal dissection for early gastric neoplasms: Korean ESD Study Group multicenter study. Gastrointest Endosc 2009; 69: 1228-35.

3. Kim JJ, Lee JH, Jung HY, Lee GH, Cho JY, Ryu CB, et al. EMR for early gastric cancer in Korea: a multicenter retrospective study. Gastrointest Endosc 2007; 66: 693-700.

4. Sasaki T, Tanabe S, Ishido K, Azuma M, Katada C, Higuchi K, et al. Recommended sedation and intraprocedural monitoring for gastric endoscopic submucosal dissection. Dig Endosc 2013; 25 Suppl 1: 79-85.

5. Yoo YC, Park CH, Shin S, Park Y, Lee SK, Min KT. A comparison of sedation protocols for gastric endoscopic submucosal dis- 
section: moderate sedation with analgesic supplementation vs analgesia targeted light sedation. Br J Anaesth 2015; 115: 84-8.

6. Sasaki T, Tanabe S, Azuma M, Sato A, Naruke A, Ishido K, et al. Propofol sedation with bispectral index monitoring is useful for endoscopic submucosal dissection: a randomized prospective phase II clinical trial. Endoscopy 2012; 44: 584-9.

7. Yamashita K, Shiwaku H, Ohmiya T, Shimaoka H, Okada H, Nakashima R, et al. Efficacy and safety of endoscopic submucosal dissection under general anesthesia. World J Gastrointest Endosc 2016; 8: 466-71.

8. Kim GE, Lee SH, Chung IS, Lee E, Shin HS, Choi JW. Severe respiratory depression precipitated by unrecognized gastric per- foration during endoscopic submucosal dissection under deep sedation - a case report -. Anesth Pain Med 2017; 12: 402-7.

9. Yagi Kuwata N, Gotoda T, Suzuki S, Mukai S, Itoi T, Moriyasu F. Reasonable decision of anesthesia methods in patients who underwent endoscopic submucosal dissection for superficial esophageal carcinoma: a retrospective analysis in a single Japanese institution. Turk J Gastroenterol 2016; 27: 91-6.

10. Bona D, Saino GI, Medda M, Panizzo V, Micheletto G. Cardiac tamponade occurred after endoscopic submucosal dissection: conservative management of the esophagopericardial fistula. Clin Case Rep 2017; 5: 1966-9. 